\title{
Ground Penetrating Radar for road engineering
}

\author{
Johannes Hugenschmidt \\ Department Road Engineering/Sealing Components, Swiss Federal Laboratories for Materials Testing and Research (EMPA), \\ CH-8600 Dibendorf, Suitzerland
}

\section{INTRODUCTION}

In recent years, Ground Penetrating Radar (GPR) has become an important tool for the inspection of roads and related structures such as bridges and tunnels. When compared to traditional methods, GPR offers several advantages. In particular, it is non-destructive, results are quasicontinuous and data can be acquired at high rates. When inspecting road pavements, traffic obstruction can be minimized or avoided.

The Road Engineering/Sealing Components Section is investigating roads and related structures using GPR in both pilot studies and contract work.

\section{GPR PRINCIPLES}

GPR is an electronnagnetic reflection method. A signal is emitted via an antenna into the structure under inspection (Fig. 1). Reflected energy caused by changes in material properties is recorded and analyzed.

Different antenna types emitting and recording different ranges of frequencies are available. Using high frequency antennas results in high resolution data but reduces the depth of penetration. Low frequency antennas provide greater depth of penetration at the expense of lower resolution.

A comprehensive overview over GPR principles can be found in [1].

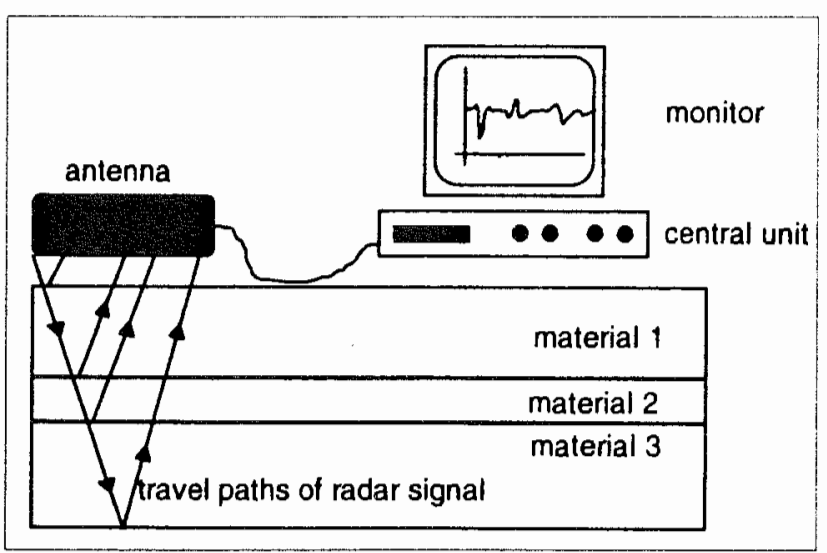

Fig. 1 - Radar principles.

\section{DATA ACQUISITION}

When inspecting roads or bridge decks, EMPA's mobile system is used. In Fig. 2, horn antennas mounted to the inspection vehicle are shown. Another antenna $(900 \mathrm{MHz})$ is mounted at the rear of the vehicle. Inside, there is a GSSI SIR-10 radar system. Using this mobile system, long road sections can be inspected within short periods of time.

Depending on the problem under investigation, vehicle speed during data acquisition is as high as 80 $\mathrm{km} / \mathrm{h}$. Thus, traffic flow obstruction can be kept to a minimum. A detailed description of typical acquisition parameters is given in [2].

Where the mobile system cannot be used, for example when inspecting tunnel walls, antennas are moved manually.

In most cases, data are stored on tape without any on-site processing applied.

\section{DATA PROCESSING AND INTERPRETATION}

Data recorded during a radar survey are copied to a PC for processing. Numerous methods are available for both the enhancement of the signal/noise ratio and interpretability. Where applicable, radar data are transferred into the coordinate system used by local authorities.

During interpretation, reflections contained within radar data are related to subsurface structures. For per-

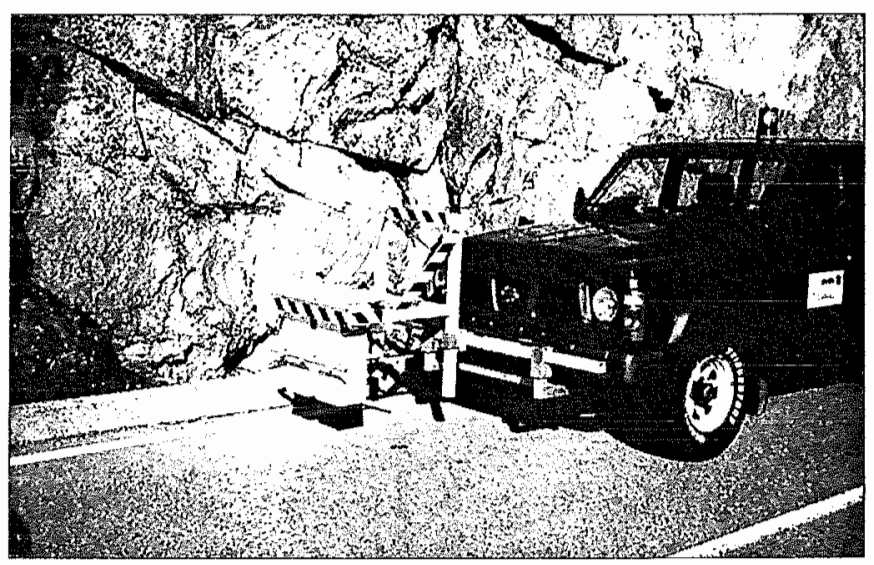

Fig. 2 - EMPA's mobile system with mounted horn antennas. 


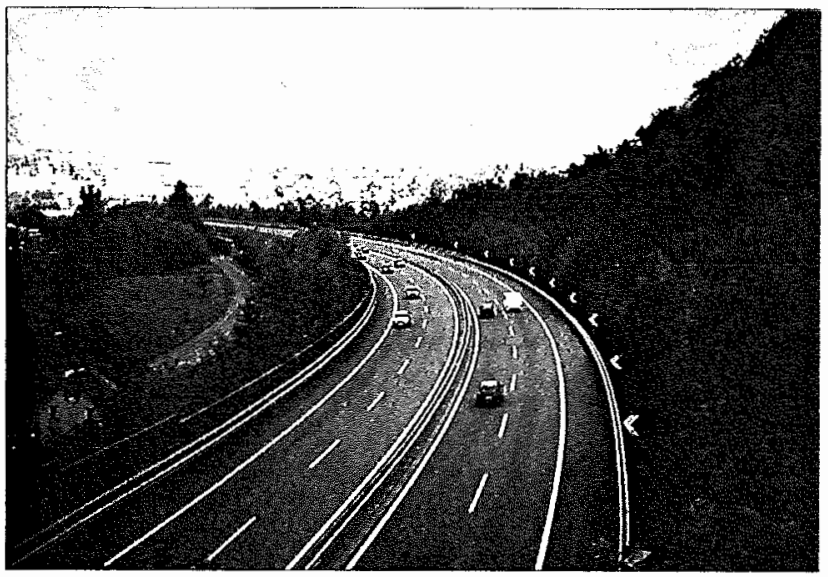

Fig. 3 - Photographic view of A2, Fluelen-Seelisberg. using the horn antennas on both lanes in both directions whilst travelling at $50 \mathrm{~km} / \mathrm{h}$. The motorway was open for traffic during data acquisition.

Radar data (after processing) of a $500 \mathrm{~m}$ long section are presented in Fig. 4 (top). Several reflections can be distinguished. The interpreted result is presented at the bottom of Fig. 4. Here, reflections visible in the dataset were related to layer boundaries obtained from cores. Thus, the overall thickness of the asphalt pavement, as well as the depth of the boundary between surface course and asphalt base course, were defined.

On the bridge, several reflections beneath the asphalt base reflection are visible in the dataset. These reflections were not interpreted because this radar inspection aimed at the investigation of the asphalt pavement.

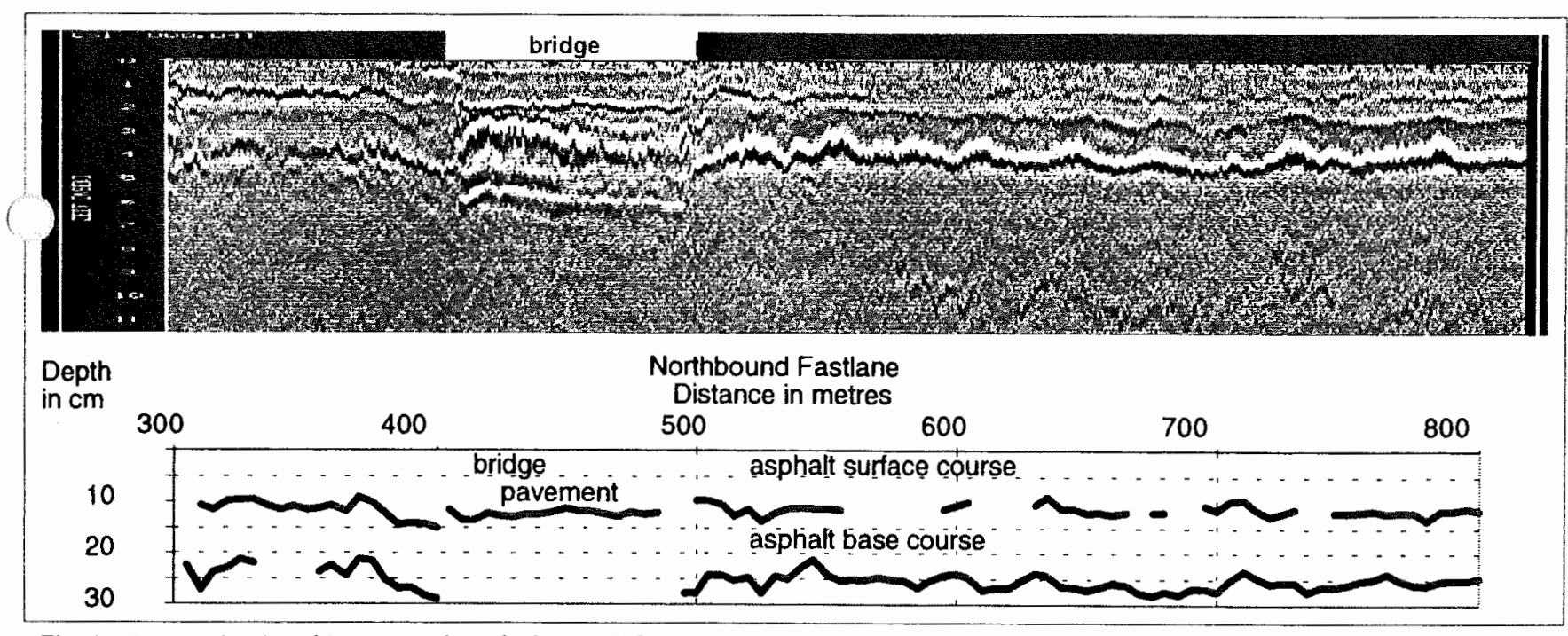

Fig. 4 - Dataset (top) and interpreted result (bottom) from Motorway A2.

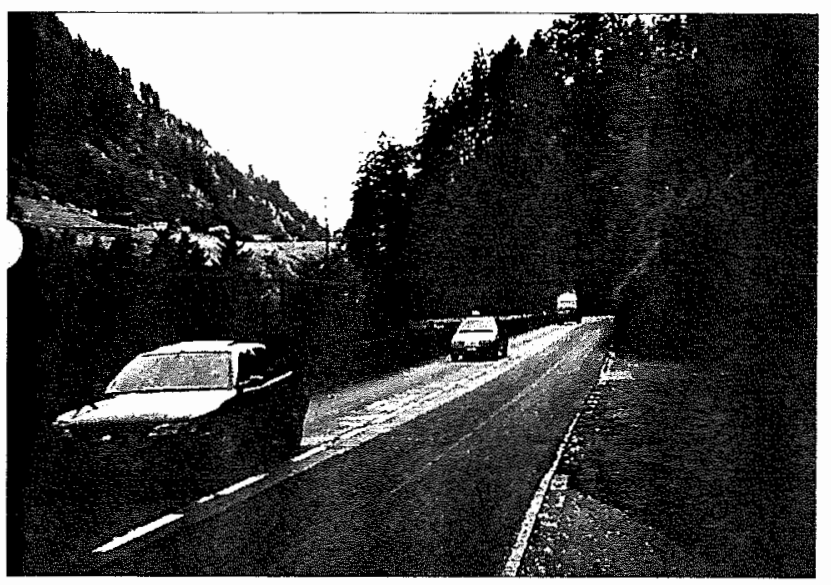

Fig. 5 - Photographic view of Gothard Road.

forming this step, additional information obtained by other methods is either useful or a necessity depending on the complexity of the problem under investigation.

\section{EXAMPLE: MOTORWAY INSPECTION}

A $3.3 \mathrm{~km}$ long section of Switzerland's National Motorway A2 (Fig. 3) was inspected. Data were acquired
However, GPR is also a powerful tool for the inspection of bridges, particularly bridge decks, as described in [3].

\section{EXAMPLE: RURAL ROAD INSPECTION (GOTTHARD ROAD)}

Gotthard Pass has been an important North-South route for many centuries. Large sections of the road leading to this pass (Fig. 5) have been rebuilt and repaired many times. Therefore, a much more complicated pavement structure than in the above example can be expected.

In Fig. 6, a 400 metre long section of the dataset acquired on the stretch shown in Fig. 5 is presented. A complex pattern of reflections is visible. Obviously the buildup of the pavement in this section is very heterogeneous.

In two locations marked as $A$ and $B$ in the dataset, interpretable reflections do not exist. This is probably due to the fact that the former gravel road has been treated with bitumen or tar in order to prevent dust, leading to a smooth transition between asphalt pavement and subbase.

In other locations such as $\mathrm{C}$, it cannot be decided which reflection is related to the asphalt pavement base without information obtained by other methods such as coring. 


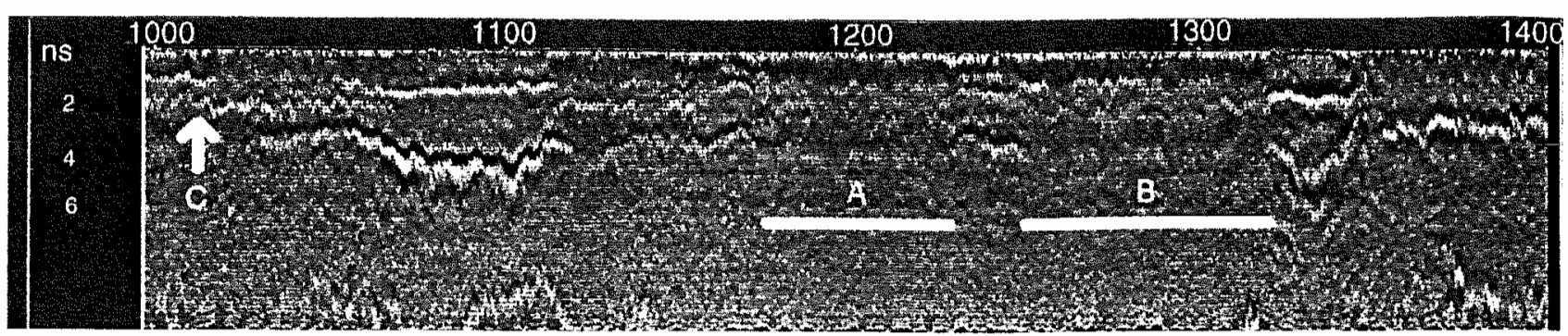

Fig. 6 - Dataset from section shown in Fig. 5.

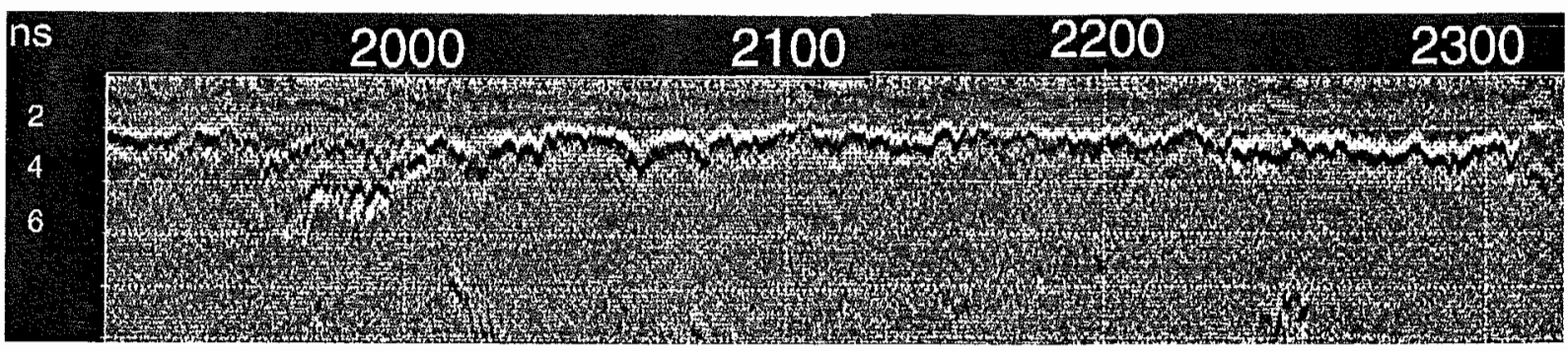

Fig. 7 - Dataset from Gothard Road.

Fortunately, the section presented in Fig. 6 was not typical for the survey. In most locations the pavement structure was less complicated. An example is shown in Fig. 7. One prominent reflection caused by the asphalt pavement base can be followed consistently throughout the section presented. Thus, using GPR it was possible to define the asphalt pavement thickness in most locations.

\section{CONCLUSIONS}

Ground Penetrating Radar is an effective tool for non-destructive testing of road pavements.

Data can be acquired efficiently using a mobile system, thus minimising traffic flow obstruction.

Additional information, such as cores, is essential in many cases. This information can be used for depth calibration. In complex environments, it is required for relating reflections in radar data to subsurface structures.

In order to exploit and increase the benefits of GPR, further practice orientated research is required. EMPA intends to play an active role in this research.

\section{ACKNOWLEDGEMENTS}

The author wishes to express his gratitude to Canton Uri, Building \& Construction Department, for the permission to publish the results. Special thanks to Mr. H. de Witte for his support and cooperation.

\section{REFERENCES}

[1] Daniels, D. J., 'Surface-Penerrating Radar' (The Institution of Electrical Engineers, London, UK, 1996).

[2] Hugenschmidt, J., Partl, M. N. and de Witte, H., 'GPR inspection of a mountain motorway - A case study', 6th International Conference on Ground Penetrating Radar, Sendai, Japan, 1996.

[3] Hugenschmidt, J., 'Non-destructive evaluation of bridge decks using GPR - Benefits and limits', US-Canada-Europe Workshop on Bridge Enginecring, Zurich, Switzerland, 1997. 